\title{
Urinary NGAL in Premature Infants
}

\author{
ADRIAN P. LAVERY, JAREEN K. MEINZEN-DERR, EDWARD ANDERSON, QING MA, MICHAEL R. BENNETT, \\ PRASAD DEVARAJAN, AND KURT R. SCHIBLER
}

\author{
Division of Neonatology [A.P.L., K.R.S.], Center for Epidemiology and Biostatistics [J.K.M.-D.], Division of Nephrology and Hypertension \\ [Q.M., M.R.B., P.D.], Cincinnati Children's Hospital Medical Center, Cincinnati, Ohio 45229; Department of Nursing [E.A.], Good \\ Samaritan Hospital, Cincinnati, Ohio 45220
}

\begin{abstract}
Premature infants are at unique risk for developing acute kidney injury (AKI) due to incomplete nephrogenesis, early exposure to nephrotoxic medications, and coexisting conditions such as patent ductus arteriosus (PDA) and respiratory distress syndrome (RDS). Unfortunately, laboratory testing for the diagnosis of AKI in this population is problematic because of the physiology of both the placenta and the extra-uterine premature kidney. Recent research has led to the development of promising biomarkers for the early detection of AKI in children but there are no published reports in neonates. Our goal was to determine whether urine neutrophil gelatinaseassociated lipocalin (NGAL) was detectable in premature infants and to correlate levels with gestational age, birth weight (BW), or indomethacin exposure. We enrolled 20 infants in four BW groups: 500-750, 751-1000, 1001-1250, and 1251-1500 g. Urine was collected every day for the first $14 \mathrm{~d}$ of life. Neonates born at earlier gestational ages and lower BWs had higher urine NGAL levels $(p<$ 0.01 ). We conclude that urine NGAL is easily obtained in premature infants and that it correlates significantly with both BW and gestational age. The use of urinary NGAL as a biomarker of AKI in premature infants warrants further investigation. (Pediatr Res 64: 423-428, 2008)
\end{abstract}

$\mathrm{T}_{\mathrm{t}}^{\mathrm{h}}$ he care of preterm infants has improved dramatically over the last few decades and has resulted in improved survival, particularly in the very low birth weight population (VLBW) defined as birth weight (BW) less than $1500 \mathrm{~g}$. Advances in mechanical ventilation, regionalization of neonatal intensive care units and use of medications such as antenatal steroids and postnatal surfactant have reduced both morbidity and mortality in this vulnerable population. However, similar advances in the diagnosis and treatment of acute kidney injury (AKI) in the preterm infant have not been realized. As the percentage of VLBW survival has increased, there has been an increase in the number of infants experiencing AKI (1).

Currently, determination of serum creatinine is the most commonly used clinical measure of neonatal glomerular function shortly after birth (2). This is a poor marker of renal

Received April 17, 2008; accepted May 13, 2008.

Correspondence: Kurt R. Schibler, M.D., Cincinnati Children's Hospital Medical Center, 3333 Burnet Avenue, Cincinnati, OH 45229; e-mail: kurt.schibler@cchmc.org Dr. Devarajan is supported by grants from the NIH/NIDDK (RO1-DK53289, P50DK52612, R21-DK070163), a Grant-in-Aid from the American Heart Association Ohio Valley Affiliate, and a Translational Research Initiative Grant from Cincinnati Children's Hospital Medical Center. Abbott Diagnostics has signed an exclusive licensing agreement with Cincinnati Children's Hospital and Columbia University for developing urine NGAL as a biomarker of acute renal failure. Dr. Schibler and the Neonatal Network Research Coordinators are supported by a grant from NIH/NICHD (U10-HD027853). function in preterm newborns because, for several days after birth, serum creatinine in the infant reflects maternal renal function due to placental transfer. Over the first few weeks of life, high serum creatinine levels are commonly observed in premature infants (3). Furthermore, there is a transient increase in serum creatinine with a peak by the fourth day of life in the preterm infant (4). For this unique population, persistently elevated serum creatinine levels confirm the diagnosis of AKI. In addition to developmental changes in kidney physiology, the administration of certain medications such as aminoglycosides and nonsteroidal antiinflammatory drugs can precipitate AKI. For example, indomethacin, which is commonly used for prophylaxis against intraventricular hemorrhage and for medical treatment of a patent ductus arteriosus (PDA), is a known risk factor for the development of AKI.

The reported incidence of neonatal AKI ranges from $6 \%$ to $24 \%(2,5)$. A recent, single-center study reported a $10-\mathrm{y}$ incidence of renal failure in $<1000 \mathrm{~g}$ infants of $5.5 \%$. Their overall mortality rate for this group was $24 \%$ (6). In a retrospective review of 20 VLBW patients diagnosed with AKI in the neonatal period, four went on to develop chronic renal insufficiency and five progressed to end-stage renal disease. At a mean follow-up age of $7.5 \mathrm{y}$, all 20 infants experienced stunted linear growth (6).

The application of functional genomics to human and animal models of AKI has led to the discovery of novel gene products that have utility as biomarkers $(7,8)$. One in particular, neutrophil gelatinase-associated lipocalin (NGAL) was recently identified as one of the earliest and most robustly induced proteins by the kidney after ischemic injury (9-13). Importantly, NGAL in the urine and plasma were found to be early predictive biomarkers of AKI in patients after surgical procedures such as cardiopulmonary bypass (14) and kidney transplantation (15), or early in the course of kidney injury associated with contrast administration (16), hemolyticuremic syndrome (17), lupus nephritis (18), pediatric intensive care (19), and chronic kidney disease (20).

NGAL has not, however, been evaluated as a potential biomarker for premature infants. This is especially important since animal studies have demonstrated that NGAL plays a

\footnotetext{
Abbreviations: AKI, acute kidney injury; BW, birth weight; NEC, necrotizing enterocolitis; NGAL, neutrophil gelatinase-associated lipocalin; PDA, patent ductus arteriosus; RDS, respiratory distress syndrome; UOP, urine output; VLBW, very low birth weight
} 
critical role in kidney development during the conversion of progenitor cells into epithelia and tubules (21). We therefore hypothesized that NGAL would be detectable in the urine of VLBW infants with ongoing nephrogenesis. Furthermore, the ability of biomarkers such as NGAL to detect AKI in this vulnerable population would enable clinicians to modify therapies before onset of oliguria or rise in serum creatinine.

The early detection of AKI in VLBW infants would enable clinicians to modify fluid administration, lower potassium concentrations in parenteral nutrition, adjust the dose or frequency of nephrotoxic medications, and initiate the use of diuretics to avoid an oliguric state. Similarly, in animal studies, renal injury can be prevented but must be detected early after the insult, certainly before the rise in serum creatinine $(21,22)$. In the current study, we sought to determine whether urine NGAL was detectable in premature infants and to correlate levels with gestational age, $\mathrm{BW}$, or indomethacin exposure.

\section{METHODS}

We conducted a prospective study to enroll 20 premature infants with BWs between 500 and $1500 \mathrm{~g}$ at three NICUs in Cincinnati, Ohio (University Hospital, Good Samaritan Hospital, and Cincinnati Children's Hospital). The Institutional Review Board at each of the three hospitals reviewed and approved the study and informed consent documents. Infants were included if their BW was between 500 and $1500 \mathrm{~g}$ and parental consent was granted. Five patients were prospectively stratified into each of the following four BW categories: $500-750,751-1000,1001-1250$, and 1251-1500 g. Infants were excluded if a significant congenital anomaly was identified.

Maternal data collected included maternal medical conditions, pregnancy complications, medications, and mode of delivery. Infant data recorded included delivery room resuscitation course, gestational age by best obstetrical estimate, daily weights, urine output (UO), total fluid administration, medications, and pertinent clinical laboratory results such as serum electrolytes, blood urea nitrogen and creatinine. Results of head ultrasounds, echocardiograms, and renal ultrasounds also were recorded. Duration of mechanical ventilation and infant medications in the first $14 \mathrm{~d}$ of life were recorded.

Urine was collected from the diaper using a cotton ball, or bladder catheter if present, within the first $24 \mathrm{~h}$ of life and daily until day of life 14 . The infant's diaper was checked every $4 \mathrm{~h}$ and collected urine was transferred to a refrigerator located at each study site. Urine samples were relocated to a $-80^{\circ}$ freezer within $48 \mathrm{~h}$ of collection.

NGAL analysis by ELISA. The urine NGAL ELISA was performed using a commercially available assay (NGAL ELISA Kit 036; AntibodyShop, Grusbakken, Denmark) that specifically detects human NGAL. The assay was performed as per the manufacturer's protocol. Briefly, $100 \mu \mathrm{L}$ of NGAL standards or diluted samples were applied onto the precoated microwells in duplicates. Microwells were then incubated for $1 \mathrm{~h}$ at room temperature and then washed with washing buffer. In succession, biotinylated NGAL antibody and HRP-streptavidin were incubated in the wells for $1 \mathrm{~h}$ each with shaking at $200 \mathrm{rpm}$. TMB substrate was added for $10 \mathrm{~min}$ in the dark before adding stop solution. Finally, NGAL concentration was measured at $450 \mathrm{~nm}$ wavelength in each well with reference reading at $620 \mathrm{~nm}$ in blank wells. The intraassay coefficients of variation were $2.1 \%$ (range: $1.3-4.0 \%$ ) in urine. Interassay variation was $9.1 \%$ (range: $6.8-18.1 \%$ ) and $8.2 \%$ (range: $2.2-$ $11.2 \%$ ) in urine. Urine creatinine was measured using a quantitative colorimetric Microplate Assay Kit (Oxford Biomedical Research, Oxford, MI) to standardize urinary NGAL for changes in urine concentration. Urinary NGAL excretion is presented as the amount of urinary NGAL in ng per mL urine as well as in ng per mg of urine creatinine to correct for differences in NGAL due to urine dilution. All measurements were made in triplicate. The laboratory investigators were blinded to the sample sources and clinical outcomes until the end of the study.

Statistical analysis. Descriptive data were reported as medians with ranges. Because urinary NGAL concentrations did not follow a normal distribution, absolute NGAL concentrations were log transformed for the purpose of the analysis. Log transformation was conducted to meet the assumptions of normality required by some of the statistical procedures. Pearson correlation coefficients were reported on log-transformed values. Differences in NGAL concentrations across BW categories were tested using one-way ANOVAs. Posthoc tests were conducted to investigate significant differences between groups using Tukey's Studentized Range Test. A repeated measures model, using generalized estimating equations, was created to investigate the change in NGAL over time. Results of the models were reported as beta coefficients $(\beta)$ with standard errors (SE).

To determine the sensitivity and specificity of NGAL in identifying markers of renal insufficiency, $2 \times 2$ tables were created. An NGAL cutoff that was $>1 \mathrm{SD}$ above the mean for each BW category was considered as a "positive" test result. The outcomes used that indicated renal insufficiency were $\mathrm{UO} \leq 1 \mathrm{~mL} / \mathrm{kg} / \mathrm{h}$ and serum creatinine $>1.2$.

\section{RESULTS}

Study population (Table 1). The study cohort was comprised of 20 subjects stratified into four groups by BW: 1) $500-750,2) 751-1000,3) 1001-1250$, and 4) 1251-1500 g (Table 1). The gender of participants was $60 \%$ male and two were small for gestational age. Racial characterization of the entire cohort was $60 \%$ white and $40 \%$ nonwhite. Maternal factors leading to premature delivery of study infants included: preeclampsia $10 \%$, premature labor unresponsive to tocolysis $35 \%$, premature rupture of fetal membranes $40 \%$, chorioamnionitis $15 \%$. Fetal distress by fetal heart rate monitoring was present in $10 \%$ of the deliveries. In utero medication exposures included magnesium sulfate, indomethacin, labetalol, synthroid, and sulindac. Antimicrobial exposures include ampicillin, azithromycin, erythromycin, clindamycin, penicillin, acyclovir, and flagyl. About $70 \%$ of the infants received prenatal steroids and Caesarean section was performed in $65 \%$ of the deliveries.

Severity of illness indicators. The incidence of mortality during the study period was $5 \%(n=1)$. The sole death was attributed to necrotizing enterocolitis (NEC) occurring on d 9 of life in an infant from the 500-750 g BW group. Four infants were assigned an Apgar score of less than five at $5 \mathrm{~min}$ of age although two infants in the 750-1000 g BW group were not assigned Apgars due to home delivery and precipitous vaginal delivery at an outlying community hospital. No infants in this study were diagnosed with hypoxic-ischemic encephalopathy. Four (20\%) of infants were diagnosed to have grade three or four intraventricular hemorrhage. Of these infants, none developed seizures or posthemorrhagic hydrocephalus during the study period. Hemodynamically significant PDA occurred in $5(25 \%)$ of study infants. For the purposes of this study, a hemodynamically significant PDA is defined as either moderate or large caliber by echocardiography or prompting treatment with indomethacin $(n=4)$ or surgical ligation $(n=$ 1). Eleven study infants $(55 \%)$ were diagnosed with respiratory distress syndrome (RDS) of prematurity. These infants were treated with mechanical ventilation and surfactant administration. NEC was diagnosed in one infant (5\%).

Medication exposure. Several medications with potential effects on the fetal kidney are administered to mothers hospitalized for risk of premature delivery. For example, nonsteroidal antiinflammatory medications (indomethacin or sulindac) are administered for their tocolytic properties to women experiencing preterm labor. Antibiotics including aminoglycosides are given to mothers with premature rupture of membranes or suspected chorioamnionitis. Two study infants were exposed in utero to medications with potential adverse renal effects (indomethacin). Although only three of the 20 mothers 
Table 1. Description of Study Population, Indicators of Severity and Medication Exposures

\begin{tabular}{|c|c|c|c|c|}
\hline Variables & $\begin{array}{c}\text { Group 1 } \\
\text { Birthweight } \\
500-750 \mathrm{~g} \\
n=5\end{array}$ & $\begin{array}{c}\text { Group 2 } \\
\text { Birthweight } \\
751-1000 \mathrm{~g} \\
n=5\end{array}$ & $\begin{array}{c}\text { Group } 3 \\
\text { Birthweight } \\
1001-1250 \mathrm{~g} \\
n=5\end{array}$ & $\begin{array}{c}\text { Group 4 } \\
\text { Birthweight } \\
1251-1500 \mathrm{~g} \\
n=5\end{array}$ \\
\hline Birthweight in grams & 616 & 948 & 1170 & 1420 \\
\hline Median (range) & $(510-742)$ & $(751-953)$ & $(1040-1230)$ & $(1335-1470)$ \\
\hline Gestational Age in wks & 25 & 27 & 30 & 30 \\
\hline Median (range) & $(24-26)$ & $(25-28)$ & $(27-30)$ & $(29-36)$ \\
\hline Male, $n(\%)$ & $2(40 \%)$ & $2(40 \%)$ & $4(80 \%)$ & $4(80 \%)$ \\
\hline Non-White Race, $n(\%)$ & $3(60 \%)$ & $3(60 \%)$ & $2(40 \%)$ & 0 \\
\hline \multicolumn{5}{|l|}{ Severity Indicators } \\
\hline Death, $n(\%)$ & $1(20 \%)$ & 0 & 0 & 0 \\
\hline Apgar at $5 \min <5, n(\%)$ & $2(40 \%)$ & 0 & $2(40 \%)$ & 0 \\
\hline Intracranial hemorrhage (Grade 3 or 4 ) & $2(40 \%)$ & $1(20 \%)$ & $1(20 \%)$ & 0 \\
\hline $\begin{array}{l}\text { PDA (moderate or large by } \\
\text { echocardiography) }\end{array}$ & $2(40 \%)$ & $1(20 \%)$ & $1(20 \%)$ & $1(20 \%)$ \\
\hline $\begin{array}{l}\text { RDS (Surfactant and/or mechanical } \\
\text { ventilation) }\end{array}$ & $5(100 \%)$ & $3(60 \%)$ & $3(60 \%)$ & 0 \\
\hline $\mathrm{NEC}, n(\%)$ & $1(20 \%)$ & 0 & 0 & 0 \\
\hline \multicolumn{5}{|l|}{ Medication Exposure } \\
\hline $\begin{array}{l}\text { Indomethacin, } n(\%) \\
\quad \text { (treatment for PDA) }\end{array}$ & $2(40 \%)$ & 0 & $1(20 \%)$ & $1(20 \%)$ \\
\hline Indomethacin, $n(\%)$ (prophylaxis for IVH) & $3(60 \%)$ & $4(80 \%)$ & 0 & 0 \\
\hline
\end{tabular}

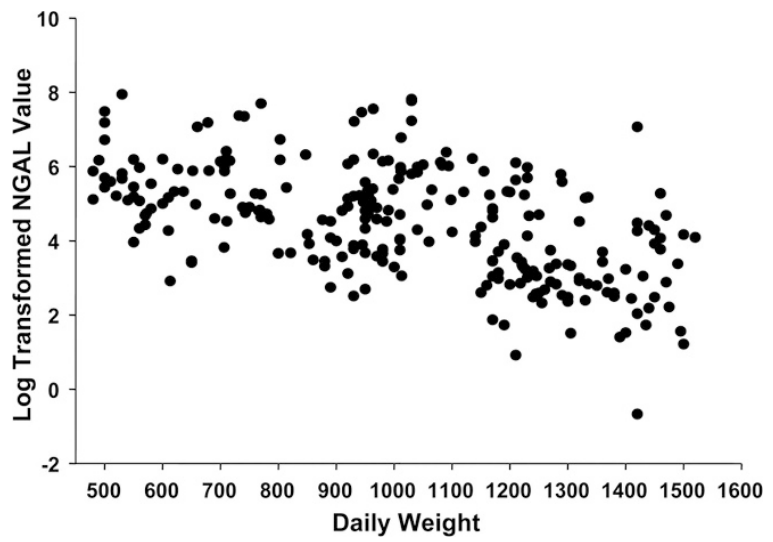

Figure 1. Log transformed absolute urinary NGAL concentration by daily weight.

were diagnosed with chorioamnionitis, 14 of the 20 mothers received antibiotics before delivery. The most common antibiotics were ampicillin and azithromycin; aminoglycosides were not administered to any mothers of study infants.

It is not uncommon for premature infants to receive medications that have renal side effects. Extremely premature infants are frequently given indomethacin for prophylaxis of intraventricular hemorrhage or for treatment of hemodynamically significant PDA. Ten study infants received indomethacin. Subsequently, two of these infants developed oliguria defined as a 24-h period with UO less than $1 \mathrm{~mL} / \mathrm{kg} / \mathrm{h}$. No infants were diagnosed with culture-proven sepsis during the study period; however, eight infants received empiric antibiotic therapy, ampicillin and gentamicin, for $5 \mathrm{~d}$ or longer for suspected infection.

NGAL is detectable in urine at birth (Fig. 1). Urine specimens were collected from 20 subjects with a BW less than or equal to $1500 \mathrm{~g}$ during the first 14-d of life. A total of 253 specimens of a possible 267 specimens $(95 \%)$ were
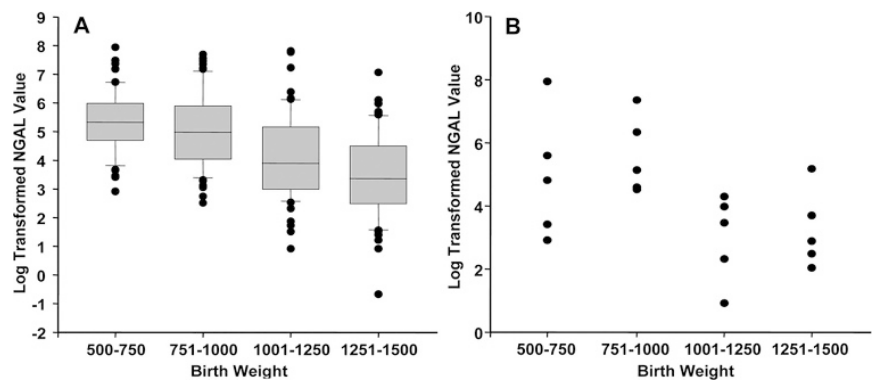

Figure 2. $A$ : Log transformation of all urinary NGAL levels $(\mathrm{ng} / \mathrm{mL})$ by birth weight category. $B$ : First urinary NGAL measurements $(\mathrm{ng} / \mathrm{mL})$.

collected using the cotton ball method. One small for gestational age subject was discharged to home on day of life six and another subject died on day of life nine. Previous studies in the laboratory confirmed that NGAL does not adsorb to the cotton [Devarajan, unpublished]. Urinary NGAL was detectable in all specimens collected from all study participants (median [range] levels of all specimens 99.2 [0.51, 2815.7]; mean \pm SD of all specimens, $244 \pm 427 \mathrm{ng} / \mathrm{mL}$ ). Urinary NGAL concentrations were negatively correlated with BW $(r=-0.53, p<0.01)$. Urinary NGAL concentrations were not correlated with postnatal day $(r=-0.09, p=0.16)$. Correction of urinary NGAL measurements with urine creatinine to account for urine concentration did not significantly influence the relationships between urinary NGAL and BW, indomethacin exposure, and oliguria; therefore, results of our analyses are reported using absolute urinary NGAL concentrations.

NGAL is inversely related to BW (Figs. 2A and 2B). Figure $2 A$ illustrates an inverse relationship between urinary NGAL concentration and BW category. According to the posthoc tests, urine NGAL concentrations from patients in BW categories 500-750 and 751-1000 were significantly different from both of the higher categories (posthoc Tukey's 
$p<0.05)$, but not different from each other. NGAL concentrations taken at the first time point (Fig. 2B) also show a similar difference on either side of the $1 \mathrm{~kg} \mathrm{BW}$. The median (range) values of the absolute NGAL levels for the four groups respectively were as follows: $123(18.4,2815), 169.4$ (91.7, 1558.8), 31.9 (2.5, 73.5$), 17.9$ (7.7, 176.8) ng/mL.

Repeated measures models were created to investigate the change of NGAL over time. BW category was included in all models. As seen in Table 2, results from the repeated measures model indicate that increasing BW category was significantly associated with a decrease in NGAL concentrations $(\beta=$ -0.46 , SE 0.16, $p=0.01)$. However, no effect was detected of postnatal day on the level of NGAL concentration in the urine $(\beta=-0.03$, SE $0.03, p=0.32)$. Inclusion of indomethacin exposure in the model did not show a statistically significant increase in NGAL concentrations among infants exposed ( $\beta=0.67, p=0.07$ ). This study included too few infants to determine whether the trend toward higher NGAL levels in exposed infants was influenced by indomethacin or by other factors associated with prematurity such as RDS, IVH, sepsis or aminoglycoside exposure. Furthermore, the timing and total dose of indomethacin were influenced by the indication for administration; IVH prophylaxis or treatment of a hemodynamically significant PDA.

NGAL as a biomarker of $\boldsymbol{A} \boldsymbol{K I}$. Individual infant NGAL data are summarized by BW categories (Fig. 3). Narrowing of NGAL levels appeared to be inversely related to BW category. The higher BW infants have narrower NGAL ranges, which

Table 2. Repeated Measures Model

\begin{tabular}{lcc}
\hline & $\beta$ Coefficient & $p$ \\
\hline Day & -0.03 & 0.31 \\
Birth weight categories & -0.46 & 0.01 \\
Indomethacin & 0.67 & 0.07 \\
\hline
\end{tabular}

may correspond to progressive maturation of the kidneys but may also be a reflection of severity of illness.

While this study was not designed to identify critical NGAL levels to predict AKI, we noticed particularly high NGAL levels in 5 patients. Oliguria and elevations in serum creatinine were noted in two of them during the first week. However, neither was noted in the remaining three.

Because of the small numbers of infants in this study, we summarized all time points for either oliguria or serum creatinine and compared them with NGAL levels in an attempt to correlate urinary NGAL levels with clinically relevant markers for renal impairment.

Information on UO was available on 197 time points in this study. One SD above the mean absolute NGAL value for each BW category was used as an arbitrary cut off for high risk for renal impairment (equivalent to a positive screening test for possible AKI). Oliguria was present at 13 of 197 time points. The sensitivity of NGAL in detecting AKI indicated by oliguria was poor at $31 \%$ and specificity was $90 \%$ (Table 3 ). We also analyzed NGAL levels with respect to serum creatinine (Table 4). Information on serum creatinine was available on 106 time points. Again, one SD above the mean absolute NGAL value for each BW category was used as an arbitrary cut off. Serum creatinine greater than $1.2 \mathrm{mg} / \mathrm{dL}$ was present in 17 of 106 time points. The sensitivity of NGAL in detecting renal impairment as indicated by serum creatinine greater than $1.2 \mathrm{mg} / \mathrm{dL}$ was also poor at $29 \%$ and specificity was $88 \%$.

Several factors might account for this degree of insensitivity at detecting renal impairment by oliguria or elevated serum creatinine. Urinary NGAL concentrations appear to be more variable among premature infants than other populations studied to date. In addition, oliguria defined by a single daily recording of UO per $\mathrm{kg}$ per hour averaged over a $24-\mathrm{h}$ period is unlikely to be a sensitive indicator of AKI. The mechanisms
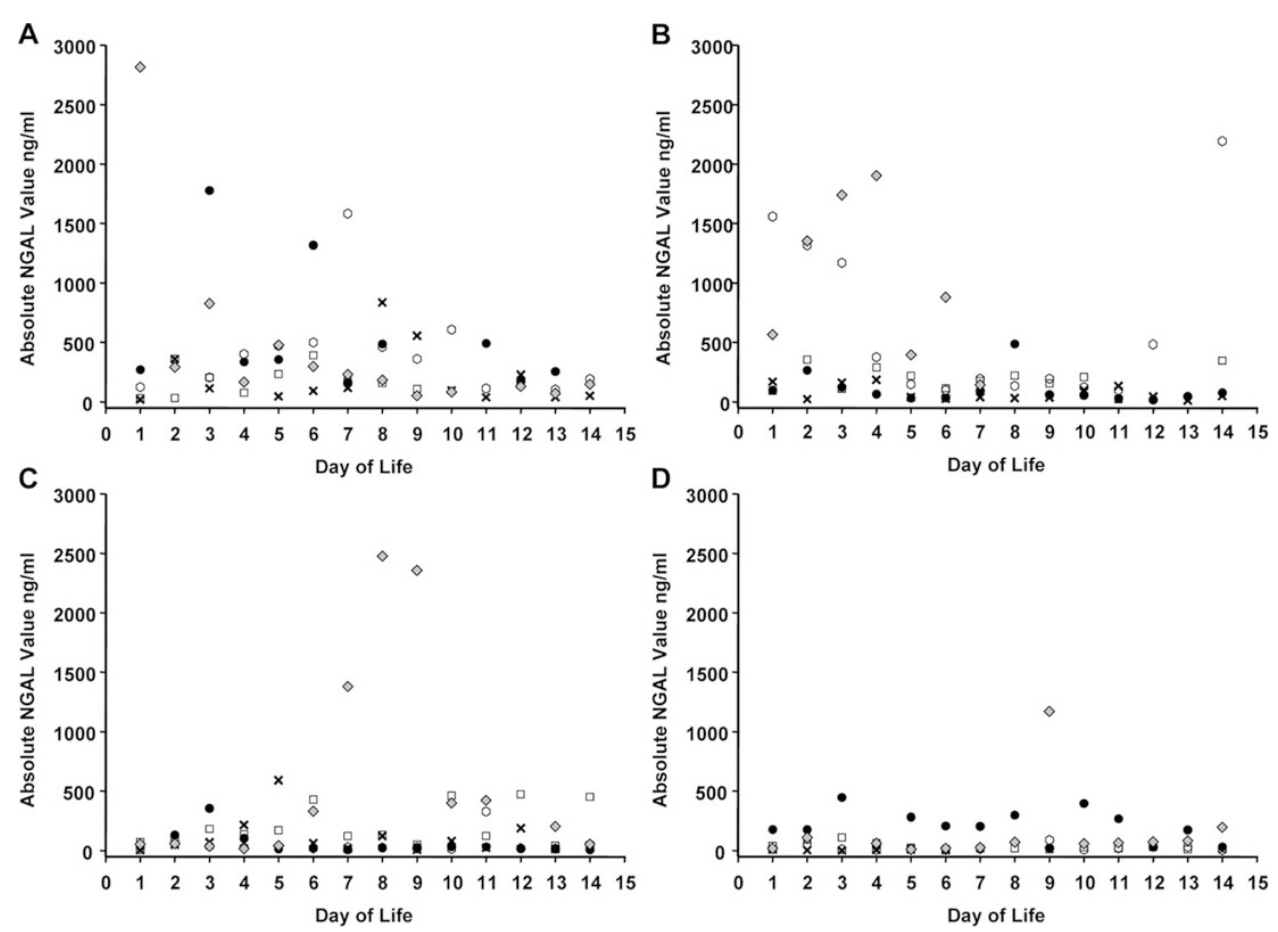

Figure 3. Absolute NGAL values (ng/ $\mathrm{mL}$ ) over the $14 \mathrm{~d}$ of life for all study infants. NGAL values for individual infants within their respective birth weight category are depicted by symbol and study identification (ID) number. $A: 500-750 \mathrm{~g}$, $B: 751-1000 \mathrm{~g}, C: 1001-1250 \mathrm{~g}, D: 1251-$ $1500 \mathrm{~g}$. 
Table 3. NGAL and Oliguria

\begin{tabular}{lccc}
\hline & \multicolumn{3}{c}{ Urine output $\leq 1 \mathrm{~mL} / \mathrm{kg} / \mathrm{h}$} \\
\hline NGAL $>1$ SD & & Yes & No \\
above mean & Yes & 4 & 19 \\
for BW & No & 9 & 165 \\
category & & & \\
\hline
\end{tabular}

Sensitivity $=31 \%$; Specificity $=90 \%$.

Table 4. NGAL and Serum Creatinine

\begin{tabular}{cccc}
\hline & \multicolumn{3}{c}{ Serum creatinine levels $>1.2 \mathrm{mg} / \mathrm{dL}$} \\
\hline NGAL $>1$ SD & & Yes & No \\
above mean & Yes & 5 & 11 \\
for BW & No & 12 & 78 \\
category & &
\end{tabular}

Sensitivity $=29 \%$; Specificity $=88 \%$.

underlying diminished UO in premature infants are variable including renal maturational events, hypovolemia, decreased renal blood flow due to shunting through a PDA, and hypoxic/ ischemic injury. Serum creatinine is likewise a poor indicator of renal impairment due to the elevated levels at birth reflecting maternal levels. The arbitrary NGAL cut off at greater than one SD might be too stringent to detect AKI as identified by oliguria or serum creatinine. Thus, to the extent that oliguria or serum creatinine reflects AKI, NGAL might prove to be useful in excluding AKI.

\section{DISCUSSION}

AKI is a relatively common problem encountered among infants delivered prematurely. Recognition of AKI in the clinical setting is likely delayed and under-appreciated due to the lack of sensitive and specific clinical indicators and diagnostic tests. When renal insufficiency is identified in the VLBW infant, it often appears to be transient. However, based upon reports in the literature documenting later recognition of chronic renal failure among children who were born prematurely, it would seem prudent to be vigilant in diagnosis and follow-up of VLBW infants who develop long-term renal complications (6). The discovery of biomarkers for AKI that might enable early recognition and clinical intervention to limit renal injury is therefore of intense contemporary interest.

Several biomarkers have been developed that detect AKI in infants and children at risk for AKI due to cardiopulmonary by-pass during cardiac surgery. Serum and urine NGAL has been demonstrated to be a sensitive and specific early marker of AKI in that patient population. Urine and serum NGAL were below the limits of detection at baseline in the majority of subjects in these studies. Among subjects with detectable NGAL levels after cardiac bypass, serum and urine NGAL measurements correlated closely (14). For the purposes of this pilot study, we examined only urine NGAL as we sought a noninvasive test for AKI.

In animal models, NGAL is expressed during the developing kidneys and its expression is extinguished once kidney development is complete. Among VLBW infants, kidney development is incomplete and continues as the infants mature. For this reason we hypothesized that NGAL would be detectable in the urine of VLBW infants and that it might decrease with increasing maturity of the infant. Indeed, we found that urine NGAL was recoverable from the diaper of premature newborns in much higher concentrations than that reported previously in older infants and children. Furthermore, an inverse relationship was observed between urine NGAL concentration and BW.

Many premature infants $(<1000 \mathrm{~g})$ receive indomethacin for IVH prophylaxis or for treatment of hemodynamically significant PDA. It is among the infants in BW categories $500-750 \mathrm{~g}$ and $750-1000 \mathrm{~g}$ that urine NGAL was highest. This study included too few infants to determine whether this association was influenced by prematurity, the presence of a hemodynamically significant PDA or indomethacin itself. To delineate this association in more detail, we performed repeated measures modeling. We found that treatment with indomethacin was not significantly associated with NGAL concentration although too few infants enrolled in this study received indomethacin to definitively prove or disprove this association. Other variables such as antibiotic exposure and duration, presence of PDA, weight loss/gain, serum electrolytes and daily total fluid administration were analyzed but showed no positive association with urine NGAL measurements. This was likely due to the small numbers of this pilot study.

The sensitivity of NGAL detecting cases of oliguria as a surrogate for AKI was poor at $31 \%$. The specificity, however, was $90 \%$, indicating that infants who do not have clinical indicators such as oliguria would test negative for AKI when using NGAL as a screening mechanism. The normal variability in urine NGAL measurements may have contributed to the diminished sensitivity of NGAL as a biomarker for AKI, but nevertheless infants who do not have clinical indicators for AKI would test negative for AKI when using NGAL as a predictive screening tool.

Urine NGAL levels in our population have shown marked variability even among similar weight groups over the first $14 \mathrm{~d}$ of life. This may be, in part, because premature kidneys are still developing and NGAL levels play an important developmental role in proliferating nephrons.

We notice that the higher BW categories have narrower NGAL ranges and that the lower BW categories took longer for their NGAL levels to narrow. Only a few time points correlate with oliguria perhaps because oliguria is not only influenced by renal function but also by daily fluid volumes, fluid restriction for PDA, or aminoglycoside administration in the first week. This makes oliguira a poor screen for kidney injury. Likewise, serum creatinine measurements in premature infants have poor predictive value and offer only confirmation of renal injury. NGAL has the potential to provide information regarding renal status before oliguria perhaps allowing the opportunity for attenuation of renal injury.

A larger study designed to provide normative NGAL data has the potential to establish threshold levels for different gestational ages and BWs. Such studies will be required to address one fundamental difference between prior NGAL studies and those in premature neonates, whether NGAL is a 
more specific biomarker of AKI than serum creatinine in this population, as has been shown for older children and adults.

Once normative values have been established, clinical application could use daily urine NGAL measurements to identify impending renal impairment. If a threshold level was attained, this would trigger appropriate reevaluation of all potentially aggravating factors including restriction of total daily fluids, alteration of aminoglycoside dosing and avoidance of indomethacin until urine NGAL levels again normalized.

Acknowledgments. We thank the Neonatal Network Research Coordinators for their assistance in study design and screening for research subjects and the nursing staffs at Good Samaritan Hospital, University Hospital, and Cincinnati Children's Hospital for assistance in the specimen procurement.

\section{REFERENCES}

1. Tommiska V, Heinonen K, Ikonen S, Kero P, Pokela ML, Renlund M, Virtanen M, Fellman V 2001 A national short-term follow-up study of extremely low birth weight infants born in Finland in1996-1997. Pediatrics 107:e2

2. Drukker A, Guignard JP 2002 Renal aspects of the term and preterm infant: a selective update. Curr Opin Pediatr 14:175-182

3. Bueva A, Guignard JP 1994 Renal function in preterm neonates. Pediatr Res 36:572-577

4. Gallini F, Maggio L, Romagnoli C, Marrocco G, Tortorolo G 2000 Progression of renal function in preterm neonates with gestational age $\leq 32$ weeks. Pediatr Nephrol 15:119-124

5. Gouyon JB, Guignard JP 2000 Management of acute renal failure in newborns. Pediatr Nephrol 14:1037-1044

6. Abitbol CL, Bauer CR, Montane B, Chandar J, Duara S, Zilleruelo G 2003 Long-term follow-up of extremely low birth weight infants with neonatal renal failure. Pediatr Nephrol 18:887-893

7. Devarajan P, Mishra J, Supavekin S, Patterson LT, Potter SS 2003 Gene expression in early ischemic renal injury: clues towards pathogenesis, biomarker discovery, and novel therapeutics. Mol Genet Metab 80:365-376
8. Nguyen MT, Ross GF, Dent CL, Devarajan P 2005 Early prediction of acute renal injury using urinary proteomics. Am J Nephrol 25:318-326

9. Supavekin S, Zhang W, Kucherlapati R, Kaskel FJ, Moore LC, Devarajan P 2003 Differential gene expression following early renal ischemia-reperfusion. Kidney Int 63:1714-1724

10. Mishra J, Ma Q, Prada A, Mitsnefes M, Zahedi K, Yang J, Barasch J, Devarajan P 2003 Identification of neutrophil gelatinase-associated lipocalin as a novel urinary biomarker for ischemic injury. J Am Soc Nephrol 14:2534-2543

11. Mishra J, Mori K, Ma Q, Kelly C, Barasch J, Devarajan P 2004 Neutrophil gelatinase-associated lipocalin (NGAL): a novel urinary biomarker for cisplatin nephrotoxicity. Am J Nephrol 24:307-315

12. Mori K, Lee HT, Rapoport D, Drexler I, Foster K, Yang J, Schmitt-Ott KM, Chen X, Li JY, Weiss S, Mishra J, Cheema FH, Markowitz G, Suganami T, Sawai K, Mukoyama M, Kunis C, D'Agati V, Devarajan P, Barasch J 2005 Endocytic delivery of lipocalin-siderophore-iron complex rescues kidney from ischemia-reperfusion injury. J Clin Invest 115:610-621

13. Schmidt-Ott KM, Mori K, Kalandadze A, Li JY, Paragas N, Nicholas T, Devarajan $\mathrm{P}$, Barasch J 2006 Neutrophil gelatinase-associated lipocalin-mediated iron traffic in kidney epithelia. Curr Opin Nephrol Hypertens 15:442-449

14. Mishra J, Dent C, Tarabishi R, Mitsnefes MM, Ma Q, Kelly C, Ruff SM, Zahedi K, Shao M, Bean J, Mori K, Barasch J, Devarajan P 2005 Neutrophil gelatinaseassociated lipocalin (NGAL) as a biomarker for acute renal injury after cardiac surgery. Lancet 365:1231-1238

15. Parikh CR, Jani A, Mishra J, Ma Q, Kelly C, Barasch J, Edelstein CL, Devarajan P 2006 Urine NGAL and IL-18 are predictive biomarkers for DGF following kidney transplantation. Am J Transplant 6:1639-1645

16. Hirsch R, Dent C, Pfriem H, Allen J, Beekman RH, Ma Q, Bennett M, Mitsnefes M, Devarajan P 2007 NGAL is an early predictive biomarker of contrast-induced nephropathy in children. Pediatr Nephrol 22:2089-2095

17. Trachtman H, Christen E, Cnaan A, Patrick J, Mai V, Mishra J, Jain A, Bullington N, Devarajan P 2006 Urinary neutrophil gelatinase-associated lipocalin in D+HUS: a novel marker of renal injury. Pediatr Nephrol 21:989-994

18. Brunner HI, Mueller M, Rutherford C, Passo MH, Witte D, Grom A, Mishra J, Devarajan P 2006 Urinary NGAL as a biomarker of nephritis in childhood-onset SLE. Arthritis Rheum 54:2577-2584

19. Zappitelli M, Washburn KK, Arikan AA, Loftis L, Ma Q, Devarajan P, Parikh CR, Goldstein SL 2007 Urine NGAL is an early marker of acute kidney injury in critically ill children: a prospective cohort study. Crit Care 11:R84

20. Mitsnefes MM, Kathman TS, Mishra J, Kartal J, Khoury PR, Nickolas TL, Barasch J, Devarajan P 2007 Serum NGAL as a marker of renal function in children with chronic kidney disease. Pediatr Nephrol 22:101-108

21. Devarajan P 2006 Update on mechanisms of ischemic acute kidney injury. J Am Soc Nephrol 17:1503-1520

22. Hewitt SM, Dear J, Star RA 2004 Discovery of protein biomarkers for renal diseases. J Am Soc Nephrol 15:1677-1689 